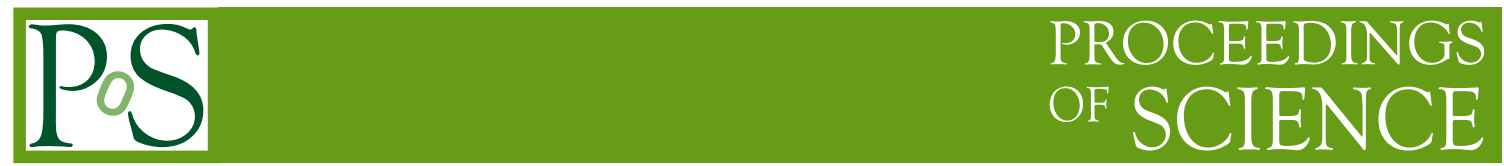

\title{
Einstein-æther gravity: a status report
}

\author{
Ted Jacobson* \\ Center for Fundamental Physics \\ University of Maryland \\ College Park, MD 20742-4111, USA \\ E-mail: jacobson@umd.edu
}

This paper reviews the theory, phenomenology, and observational constraints on the coupling parameters of Einstein-æther gravity, i.e. General Relativity coupled to a dynamical unit timelike vector field. Several new remarks and a discussion of open issues are included.

From Quantum to Emergent Gravity: Theory and Phenomenology

June 11-15 2007

Trieste, Italy

\footnotetext{
* Speaker.
} 


\section{Introduction}

There are reasons to suspect that the vacuum in quantum gravity may determine a preferred rest frame at the microscopic level. However, if such a frame exists, it must be very effectively concealed from view. Numerous observations severely limit the possibility of Lorentz violating physics among the standard model fields [1]. The constraints on Lorentz violation in the gravitational sector are generally far weaker.

To allow for gravitational Lorentz violation without abandoning the framework of general relativity (GR), the background tensor field(s) breaking the symmetry must be dynamical. Einsteinæther theory is of this type. In addition to the spacetime metric tensor field $g_{a b}$ it involves a dynamical, unit timelike vector field $u^{a}$. Like the metric, and unlike other classical fields, the unit vector cannot vanish anywhere, so it breaks local Lorentz symmetry down to a rotation subgroup. It defines a congruence of timelike curves filling all of spacetime, like an omnipresent fluid, and so has been dubbed the "æther". This paper aims to provide a review of what has been learned about this theory, the status of observational constraints, and some currently open issues.

A primary motivation for studying Einstien-æther theory— "æ-theory " for short—is the quantum gravity suspicion mentioned above. A secondary aim is to develop a viable and reasonably natural foil against which to compare gravitational observations, in an era when numerous alternate gravity theories have already been either ruled out or severely constrained. A third source of interest is the theoretical laboratory it offers for studying diffeomorphism invariant physics with preferred frame effects.

The action involving metric and æther is highly constrained by diffeomorphism invariance, locality, and the unit constraint on $u^{a}$. The only term with no derivatives is the cosmological constant, there are no terms with one derivative (other than a total divergence), and there are five terms with two derivatives,

$$
S=-\frac{1}{16 \pi G} \int \sqrt{-g}\left(R+K_{m n}^{a b} \nabla_{a} u^{m} \nabla_{b} u^{n}\right) d^{4} x .
$$

Here $R$ is the Ricci scalar, and the tensor $K_{m n}^{a b}$ is defined by

$$
K_{m n}^{a b}=c_{1} g^{a b} g_{m n}+c_{2} \delta_{m}^{a} \delta_{n}^{b}+c_{3} \delta_{n}^{a} \delta_{m}^{b}+c_{4} u^{a} u^{b} g_{m n},
$$

where the $c_{i}$ are dimensionless coupling constants. Like general relativity, pure classical æ-theory defined by this action is scale-free. (The metric signature is $(+---)$, the speed of light defined by the metric $g_{a b}$ is unity, and the æther is taken to be dimensionless.) The term $R_{a b} u^{a} u^{b}$ can be expressed as the difference of the $c_{3}$ and $c_{2}$ terms, up to a total derivative, so is not independent. In computations the unit timelike constraint on the æther is usually imposed by adding a Lagrange multiplier term $\lambda\left(g_{a b} u^{a} u^{b}-1\right)$ to the action. The covariant derivative operator $\nabla_{a}$ involves derivatives of the metric through the connection components, and the unit vector is nowhere vanishing, hence the terms quadratic in $\nabla_{a} u^{m}$ are quadratic in derivatives of both æther and metric perturbations, so the metric and æther modes are coupled. The field equations are written out in detail in many of the references, beginning with Ref. [2]. It is noteworthy that the æther stress tensor includes second derivative terms arising from the variation of the metric in the connections. In all phenomenology work to date, it has been assumed that the æther is aligned at large scales with the rest frame of the microwave background radiation. 
The restriction to no more than two derivatives is motivated by the standard precepts of effective field theory [3]: higher derivatives would be suppressed by one power of a small length scale for each extra derivative. The natural size of the coupling constants $c_{i}$ depends on unknown physics at high energies. If the Planck scale is the only relevant scale then the $c_{i}$ are naturally all of order unity. If on the other hand there is an additional scale or scales characterizing the Lorentz violating physics, then the $c_{i}$ might be naturally smaller and could differ from each other in order of magnitude. Note that although the $c_{4}$ term in the action is quartic in the æther field, it makes a quadratic contribution to the kinetic terms for metric and æther perturbations when expanded around a flat background with a constant æther of unit norm.

Einstein-æther theory is similar to the vector-tensor gravity theories studied long ago by Will and Nordvedt [4], but with the crucial difference that the vector field is constrained to have unit norm. This constraint eliminates a wrong-sign kinetic term for the length-stretching mode [5], hence gives the theory a chance at being viable. An equivalent theory using the tetrad formalism was first studied by Gasperini [6], and in the above form it was introduced by Jacobson and Mattingly [7]. Related vector-tensor gravity theories are that of Kostelecky and Samuel [8] which corresponds to the Maxwell-like special case of $æ$-theory $\left(c_{3}=-c_{1}, c_{2}=c_{4}=0\right)$, both with a fixed norm and with a symmetry breaking potential for the vector, of Gripaios [9] which has all two-derivative terms and a symmetry breaking potential for the vector, and the generalized Einstein-æther theories of Zlosnik, Ferreira and Starkman [10] and of Zhao [11], in which the æther kinetic terms of (1.1) are replaced by functions thereof. Kanno and Soda [12] introduced dependence of the coupling parameters on a scalar field that has its own dynamics, and studied inflationary cosmology for one such model. Other theories involving scalar fields in addition to a vector field with a Maxwell-like action for the vector are Bekenstein's TeVeS [13] and Moffat's STVG [14].

\subsection{Matter couplings}

Lorentz-violating (LV) effects in the matter sector are produced by couplings of the matter to the æther. (More complicated Lorentz symmetry breaking patterns require LV extensions of general relativity with other symmetry breaking fields [15].) Such effects have been highly constrained by observations [1], so if they exist they are very weak. In this review I will therefore assume that matter couples universally to the metric $g_{a b}$. This assumption is motivated by phenomenology, but goes against the precepts of effective field theory since the Lorentz violation in the gravitational sector would presumably induce Lorentz violating terms in the matter action via loop effects. Supersymmetry could conceivably provide a natural suppression of these terms, as discussed briefly in section 9.

\subsection{Metric redefinitions}

When investigating aspects of the theory that do not involve matter, it can be helpful to exploit the metric redefinition $g_{a b}^{\prime}=g_{a b}+(\zeta-1) u_{a} u_{b}$, which "stretches" the metric tensor in the æther direction by a positive factor $\zeta$. (A negative factor would return a Euclidean signature metric.) The action (1.1) for $\left(g_{a b}^{\prime}, u^{\prime a}=u^{a} / \sqrt{\zeta}\right)$ takes the same form as that for $\left(g_{a b}, u^{a}\right)$, with new coefficients $c_{i}^{\prime}$. The relation between the $c_{i}^{\prime}$ and $c_{i}$ was worked out in [16], and is conveniently given in terms of 
certain combinations with simple scaling behavior:

$$
\begin{aligned}
c_{14}^{\prime} & =c_{14} \\
c_{123}^{\prime} & =\zeta c_{123} \\
c_{13}^{\prime}-1 & =\zeta\left(c_{13}-1\right) \\
c_{1}^{\prime}-c_{3}^{\prime}-1 & =\zeta^{-1}\left(c_{1}-c_{3}-1\right) .
\end{aligned}
$$

Note that in the absence of matter couplings a one-parameter set of Einstein-æther theories is actually pure vacuum GR in disguise [17, 16]: the parameters $c_{i}^{\prime}$ all vanish if $c_{14}=c_{123}=2 c_{1}-$ $c_{1}^{2}+c_{3}^{2}=0$, and $\zeta>0$ provided $c_{13}<1$.

\section{Newtonian and post-Newtonian limits}

In the weak-field, slow-motion limit æ-theory reduces to Newtonian gravity [18], with a value of Newton's constant $G_{\mathrm{N}}$ related to the parameter $G$ in the action (1.1) by

$$
G_{\mathrm{N}}=\frac{G}{1-c_{14} / 2},
$$

where $c_{14} \equiv c_{1}+c_{4}$. (Similar notation is used below for other additive combinations of the $c_{i}$.) As long as $c_{14}<2$, the Newtonian limit is thus recovered. If $c_{14}>2$ gravity is repulsive. This suggests the possibility of resolving singularities by anti-gravity if the coupling coefficients $c_{i}$ were not constants [6]. Here I discuss only the theory with constant coefficients.

All parameterized post-Newtonian (PPN) parameters [19] of ae-theory except the preferred frame parameters $\alpha_{1,2}$ agree with those of GR for any choice of the $c_{i}$. In particular, the EddingtonRobertson-Schiff parameters $\beta$ and $\gamma$ are both unity [2], the Whitehead parameter $\xi$ vanishes [20], and the five energy-momentum conservation parameters $\alpha_{3}, \zeta_{1,2,3,4}$ vanish simply because the theory is derived from a lagrangian. The parameter $\alpha_{2}$ was found for small $c_{i}$ in [21], and the exact values of $\alpha_{1}$ and $\alpha_{2}$ were found in [20]:

$$
\begin{aligned}
& \alpha_{1}=\frac{-8\left(c_{3}^{2}+c_{1} c_{4}\right)}{2 c_{1}-c_{1}^{2}+c_{3}^{2}} \\
& \alpha_{2}=\frac{\alpha_{1}}{2}-\frac{\left(c_{1}+2 c_{3}-c_{4}\right)\left(2 c_{1}+3 c_{2}+c_{3}+c_{4}\right)}{c_{123}\left(2-c_{14}\right)}
\end{aligned}
$$

(This particular way of expressing $\alpha_{2}$ was given in [22].) In deriving the PPN parameters, the 00component of the metric perturbation and the 0 -component of the aether perturbation are expanded to $O(2)$ in the Newtonian potential, while the $0 i$-components of the metric and $i$ components of the aether are truncated at their lowest order, $O(1.5){ }^{1}$

Observations currently impose the strong constraints $\alpha_{1} \lesssim 10^{-4}$ and $\alpha_{2} \lesssim 4 \times 10^{-7}$ [19]. Since æ-theory has four free parameters $c_{i}$, we may set $\alpha_{1,2}$ exactly to zero by imposing the conditions [20]

$$
\begin{aligned}
& c_{2}=\left(-2 c_{1}^{2}-c_{1} c_{3}+c_{3}^{2}\right) / 3 c_{1} \\
& c_{4}=-c_{3}^{2} / c_{1} .
\end{aligned}
$$

\footnotetext{
${ }^{1}$ The 0 -component of the aether is expanded explicitly only to $O(1)$ in Ref. [20], but the contribution of the $O(2)$ part is implicitly incorporated by use of the identity (A.26).
} 
With $(2.4,2.5)$ satisfied, all the PPN parameters of æ-theory are equivalent to those of GR.

The parameters $\alpha_{1,2}$ can also be set to zero by imposing $c_{13}=c_{14}=0$, but this case is pathological, as discussed in section 8. Also discussed there is the case $c_{123}=0$, for which $\alpha_{2}$ can diverge. Another special case occurs when $2 c_{1}-c_{1}^{2}-c_{3}^{2}=0$, for which $\alpha_{1}$ diverges unless also $c_{3}^{2}+c_{1} c_{4}=0$ in which case it is indeterminate. Together these imply $c_{14}=2$, so Newton's constant (2.1) diverges in this case.

\section{Homogeneous isotropic cosmology}

Assuming spatial homogeneity and isotropy, $u^{a}$ necessarily coincides with the 4-velocity of the isotropic observers, and automatically satisfies its field equation. The æther stress tensor is constructed purely form the spacetime geometry and is identically conserved. It is just a certain combination of the Einstein tensor and the stress tensor of a perfect fluid with energy density proportional to the inverse square of the scale factor, like the curvature term in the Friedman equation $[23,18]$,

$$
T_{a b}^{\mathfrak{x t h e r}}=-\frac{c_{13}+3 c_{2}}{2}\left[G_{a b}-\frac{1}{6}{ }^{(3)} R\left(g_{a b}+2 u_{a} u_{b}\right)\right] .
$$

The latter contribution plays no important cosmological role since the spatial curvature is small, while the former renormalizes the gravitational constant appearing in the Friedman equation, yielding [18]

$$
G_{\text {cosmo }}=\frac{G}{1+\left(c_{13}+3 c_{2}\right) / 2} .
$$

Since $G_{\text {cosmo }}$ is not the same as $G_{\mathrm{N}}$ the expansion rate of the universe differs from what would have been expected in GR with the same matter content. The ratio is constrained by the observed primordial ${ }^{4} \mathrm{He}$ abundance to satisfy $\left|G_{\text {cosmo }} / G_{\mathrm{N}}-1\right| \lesssim 1 / 8$ [18]. When the PPN parameters $\alpha_{1,2}$ are set to zero by $(2.4,2.5)$, it turns out that $G_{\mathrm{cosmo}}=G_{\mathrm{N}}$, so this nucleosynthesis constraint is automatically satisfied [20].

\section{Linearized wave modes}

When linearized about a flat metric and constant æther, æx-theory possesses five massless modes for each wave vector: two spin-2, two spin-1, and one spin- 0 mode. The squared speeds of these modes relative to the æther rest frame are given by [24]

$$
\begin{array}{ll}
\text { spin-2 } & 1 /\left(1-c_{13}\right) \\
\text { spin-1 } & \left(2 c_{1}-c_{1}^{2}+c_{3}^{2}\right) / 2 c_{14}\left(1-c_{13}\right) \\
\text { spin-0 } & c_{123}\left(2-c_{14}\right) / c_{14}\left(1-c_{13}\right)\left(2+c_{13}+3 c_{2}\right)
\end{array}
$$

The corresponding polarization tensors were found in one gauge in Ref. [24] and in another gauge in Ref. [22]. The former gauge choice for the metric and æther perturbations $h_{a b}$ and $v^{a}$ is $h_{0 i}=0$ and $v^{i}{ }_{i}=0$. In this gauge the nonzero components of the polarization for waves in the 3-direction 
are given by

$$
\begin{array}{lll}
\text { spin-2 } & h_{12}, & h_{11}=-h_{22} \\
\text { spin-1 } & v_{I}, & h_{3 I}=\left[2 c_{14} c_{13}^{2} /\left(2 c_{1}-c_{1}^{2}+c_{3}^{2}\right)\right]^{1 / 2} v_{I} \\
\text { spin-0 } & v_{0}, \quad h_{00}=-2 v_{0}, \quad h_{11}=h_{22}=-c_{14} v_{0}, \quad h_{33}=\left[2 c_{14}\left(1+c_{2}\right) / c_{123}\right] v_{0},
\end{array}
$$

where the index $I$ takes only the transverse values 1,2 .

The energy of the spin-2 modes is always positive, while for the spin-1 modes it has the sign of $\left(2 c_{1}-c_{1}^{2}+c_{3}^{2}\right) /\left(1-c_{13}\right)$, and for the spin-0 modes it has the sign of $c_{14}\left(2-c_{14}\right)$ [25, 22]. (These reduce to the results of Ref. [26] in the decoupling limit where gravity is turned off.) Under the metric redefinition $g_{a b} \rightarrow g_{a b}+(\zeta-1) u_{a} u_{b}$, (1.3) can be used to verify that the squared speeds all scale as $1 / \zeta$ as expected, and the signs of the energy are invariant.

Several constraints are imposed by stability conditions on the linearized modes. The squared speeds correspond to (frequency/wavenumber) ${ }^{2}$, so must be non-negative to avoid imaginary frequency instabilities. They must moreover be greater than or equal to unity, to avoid the existence of vacuum Čerenkov radiation by matter [5]. (The existence of ultra high energy cosmic rays, which propagate near the metric light cone, requires that the metric and æther modes propagate at no less than an extremely small amount below the metric speed of light.) And the mode energy densities should be positive, to avoid dynamical instabilities.

With the $\alpha_{1,2}=0$ conditions $(2.4,2.5)$ imposed, the squared speeds expressed in terms of $c_{ \pm} \equiv c_{1} \pm c_{3}$ are

$$
\begin{array}{ll}
\text { spin-2 } & 1 /\left(1-c_{+}\right) \\
\text {spin-1 } & \left(c_{+}+c_{-}-c_{+} c_{-}\right)\left(c_{+}+c_{-}\right) / 4 c_{+} c_{-}\left(1-c_{+}\right) \\
\text {spin-0 } & c_{+} / 3 c_{-}\left(1-c_{+}\right),
\end{array}
$$

the sign of the spin-1 mode energy is that of $\left(c_{+}+c_{-}-c_{+} c_{-}\right) /\left(1-c_{+}\right)$, and $c_{14}=2 c_{+} c_{-} /\left(c_{+}+\right.$ $c-)$. The conditions that the squared spin- 2 and spin- 0 mode speeds be greater than or equal to unity then restrict $c_{ \pm}$to the region

$$
\begin{aligned}
& 0 \leq c_{+} \leq 1 \\
& 0 \leq c_{-} \leq c_{+} / 3\left(1-c_{+}\right) .
\end{aligned}
$$

Some algebra shows that the inequalities $0 \leq c_{+} \leq 1$ and $0 \leq c_{-}$also ensure that the squared spin1 mode speed (4.8) is also greater than or equal to unity. They also imply $0 \leq c_{14}<2$, hence the condition for attractive gravity mentioned in section 2 need not be separately imposed. Also the energies of the spin- 0 and spin- 1 modes are then positive. That is, when $\alpha_{1,2}=0$ all of the requirements for all of the modes are met and gravity is attractive if and only if and $c_{ \pm}$are restricted by the inequalities $(4.10,4.11)$ [20]. The allowed range is the shaded region in Fig. 1.

Interestingly, if the mode speeds are instead required to be less than unity (sub-luminal), then the spin- 1 and spin-0 energy densities are negative. Hence not only the Čerenkov constraint, but also energy positivity (together with $\alpha_{1,2}=0$ ) requires mode speeds greater than unity. 


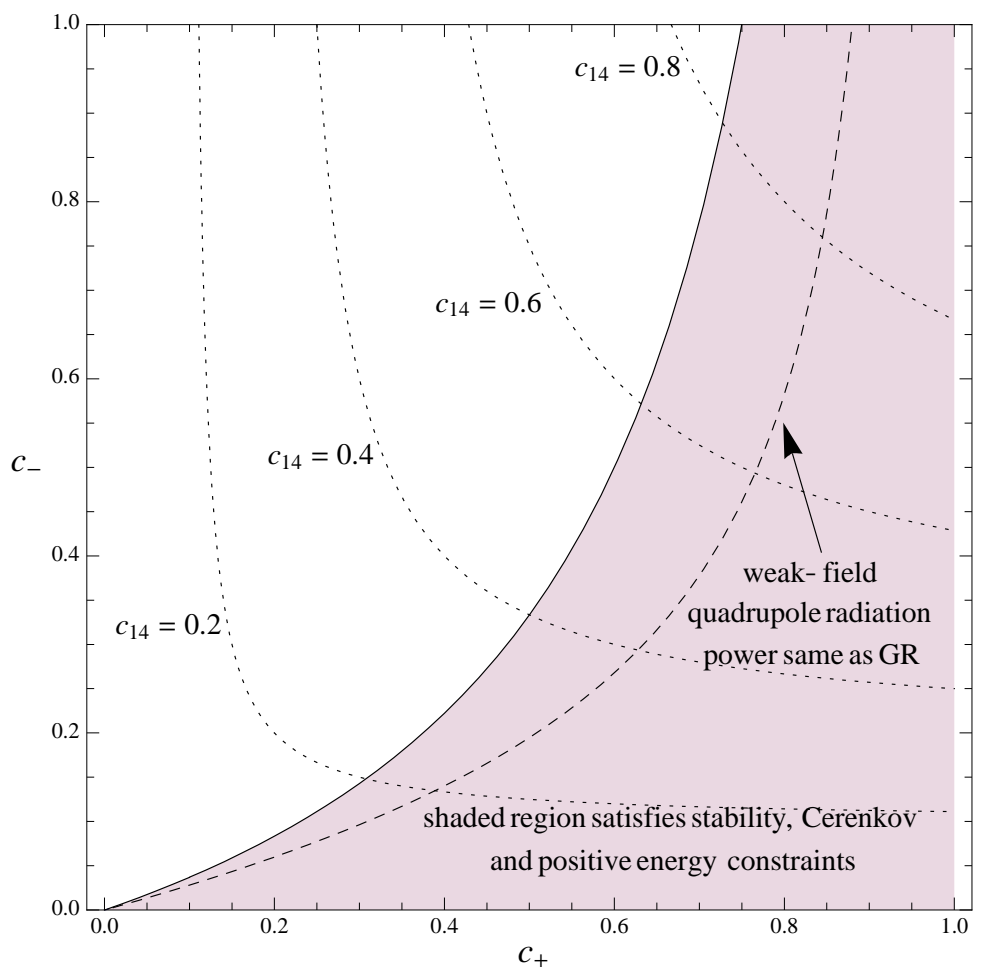

Figure 1: The shaded region satisfies the stability, Čerenkov, and positive energy constraints $(4.10,4.11)$ on $c_{ \pm}$after having imposed the vanishing of the PPN parameters $\alpha_{1,2}$ by the conditions $(2.4,2.5)$. The dashed curve is the locus where the net radiation power from a source with weak self-field matches that of GR. The four dotted curves are contours of fixed $c_{14}$.

\section{Primordial perturbations}

The behavior of primordial cosmological perturbations in ae-theory has been studied in Refs. $[26,27]$. The coupling to the æther modifies the spin-2 perturbations in two ways: it changes their propagation speed, as already discussed above for perturbations of flat space, and it changes their amplitude, since that is determined by the "bare" $G$ in the action (1.1) rather than by the measured Newton constant $G_{\mathrm{N}}$. The result is that, given the same $G_{\mathrm{N}}$, and assuming the PPN parameters $\alpha_{1,2}$ vanish, the power in these perturbations differs from that in GR by the factor $\left(1-c_{14} / 2\right)\left(1-c_{13}\right)^{1 / 2}$. When the stability constraints $(4.10,4.11)$ are satisfied this factor is smaller than unity, hence these spin-2 perturbations are even more difficult to detect than in GR.

Perturbations of the æther can be decomposed into spin- 0 and spin- 1 modes, both of which decay by themselves as the universe expands. In the presence of a scalar inflaton, however, the spin- 0 æther mode is sourced and no longer decays away. For single scalar field slow-roll inflation, it turns out that the power spectrum is generally modified, but it is unchanged when the PPN parameters vanish.

The late time evolution of the primordial perturbations is modified, since anisotropic photon and neutrino stresses in the radiation dominated epoch source the spin-1 mode. This leads to modified matter and CMB spectra [27]. The effect is rather small however, and is degenerate with 
matter-galaxy bias and with neutrino masses.

Primordial perturbations are thus difficult to distinguish in ae-theory and GR.

\section{Radiation damping and strong self-field effects}

Two-body dynamics and radiation in ae-theory have been analyzed for purely weak fields in Ref. [22], and including strong self gravity effects in Ref. [28]. In general the radiation includes not only spin- 2 waves sourced by a varying quadrupole moment, but also spin- 1 waves sourced by quadrupole and dipole moments and spin-0 waves sourced by quadrupole, dipole, and monopole moments. As seen in section 4, the waves of different spin propagate in general at different speeds relative to the æather rest frame. Direct observation of all of these waves is in principle possible with existing gravitational wave antennas [19]. With waves of sufficient amplitude from multiple sources, and a network of detectors, the æther rest frame and the wave speeds (4.1)-(4.3) and polarizations (4.4)-(4.6) could all be measured.

\subsection{Weak fields}

If the fields are weak everywhere (including inside the radiating bodies), and the PPN parameters $\alpha_{1,2}$ vanish, then the dipole source vanishes and the monopole source is uncoupled to the fields. In this regime therefore all radiation is sourced by the quadrupole, as in GR. The net power radiated in spin-0, spin-1, and spin-2 modes in this case is given by $\left(G_{\mathrm{N}} \mathscr{A} / 5\right) \dddot{Q}_{i j}^{2}$, where $Q_{i j}$ is the quadrupole moment and $\mathscr{A}=\mathscr{A}\left[c_{i}\right]$ is a function of the coupling parameters $c_{i}$ that reduces to unity in the case of GR. Agreement with the damping rate of GR (confirmed to $\sim 0.1 \%$ in binary pulsar systems [19]) can be achieved by imposing the condition $\mathscr{A}\left[c_{i}\right]=1$, which is consistent with the constraints $(4.10,4.11)$, as shown by the dashed line in Fig. 1.

\subsection{Strong field effects}

For compact sources with strong internal fields such as neutron stars or black holes, terms of higher order in $G m / d$ (where $m$ and $d$ are the mass and characteristic size of the source) can play an important role in the dynamics and radiation. Since background fields and radiation fields vary little over compact source dimensions, these strong field effects can be handled using an "effective source" dynamics specified by a worldline action integral [28]

$$
S=-m_{0} \int d \tau\left[1+\sigma\left(v^{a} u_{a}-1\right)+\sigma^{\prime}\left(v^{a} u_{a}-1\right)^{2}+\ldots\right]
$$

where $v^{a}$ is the 4-velocity of the body, $u_{a}$ is the local background value of the æther, and $\sigma$ and $\sigma^{\prime}$ are a constants characterizing the body, called a "sensitivity parameters" or just "sensitivities". A similar technique was introduced by Eardley [29] for scalar-tensor gravity, in which the first sensitivity measures the dependence of the action on the local background value of the scalar field. The ellipses in (6.1) stand for all other invariants that can be formed from the particle worldline and the background fields. All of these are of higher order in the velocity relative to the æther or involve more derivatives and are hence suppressed. Among other things, the sensitivities determine the dependence of energy on velocity, $E=m_{0}+\frac{1}{2}(1+\sigma) m_{0} v^{2}+\frac{3}{8}\left(1+\sigma-\sigma^{\prime}\right) m_{0} v^{4}+\ldots$. (This 
is obtained by expressing the action (6.1) in the form $\int d t(T-V)$ where $V=m_{0}$, and reading off $E=T+V$.) At lowest order in binding energy $\Omega, \sigma$ is given by

$$
\sigma=\left(\alpha_{1}-\frac{2}{3} \alpha_{2}\right)(\Omega / m)+\mathscr{O}\left(f\left[c_{i}\right]\left(G_{\mathrm{N}} m / d\right)^{2}\right)
$$

where $f\left[c_{i}\right]$ scales as $c_{i}$ for $c_{i} \ll 1 .^{2}$ Note that the leading term vanishes when $\alpha_{1,2}$ vanish. The higher order terms in $\sigma$ have not yet been computed, nor have even the lowest order terms in $\sigma^{\prime}$ (which will also scale as $c_{i}$ ). They presumably differ for black holes and stars, and will depend on the equation of state of the star.

Nonzero sensitivities lead to a number of phenomena that are constrained by observations, including dependence of dynamics on the ambient æther vector, modified post-Newtonian twobody dynamics, modified quadrupole sourced radiation, and both monopole and dipole sourced radiation. When $\alpha_{1,2}=0$, all of these constraints are met provided the sensitivities are less than $\sim 0.001$, which will certainly be the case if $c_{i} \lesssim 0.01$ [28]. To be more precise would require knowing the actual dependence of the sensitivities on the $c_{i}$. It is conceivable that the constraints will be much weaker.

To determine the strong field constraints just mentioned, it is necessary to make some assumption about the speed $V$ of the observed systems with respect to the background æther frame. It was argued in Ref. [28] that neglect of terms involving this speed is justified provided $V \lesssim 10^{-2}$, which is easily satisfied for any known proper motion relative to the rest frame of the microwave background radiation.

\section{Spherically symmetric stars and black holes}

Unlike GR, æ-theory has a spherically symmetric mode, corresponding to radial tilting of the æther, and there is a three-parameter family of spherically symmetric static vacuum solutions [31]. If asymptotic flatness is imposed and the mass fixed, there remains a one-parameter family [2, 31], whereas GR has the unique Schwarzschild solution. As explained below, the evidence suggests that only two solutions in this one-parameter family are astrophysically relevant, at least provided the couplings are not too large.

The total energy of an asymptotically flat solution of æ-theory receives a contribution from the asymptotic $1 / r$ falloff of the æther [25, 32]. The æther normalization condition locks this contribution to that of the metric, so the energy can be expressed in terms of the metric alone. It turns out to be just $E=r_{0} / G_{\mathrm{N}}$, where $r_{0}$ is the coefficient of the $1 / r$ term in the $g^{r r}$ inverse metric component ${ }^{3}$ and $G_{\mathrm{N}}$ is Newton's constant (2.1). That is, it agrees with the Newtonian mass in the Newtonian limit, which is inevitable given that the theory has a Newtonian limit. This is how the mass of the solutions discussed below is determined.

\footnotetext{
${ }^{2}$ This corrects an error in version 1 of Ref. [28], where $\sigma$ is said to scale as $c_{i}^{2}$. (Also the a prefactor $c_{14}$ in Eqn. (70) should be deleted.) As a result of this correction, the likely constraints on $c_{i}$ are an order of magnitude stronger, as stated here [30].

${ }^{3}$ This is the asymptotic Misner-Sharp energy [33], covariantly defined by $r_{0}=\lim _{r \rightarrow \infty}(r / 2)\left(1-g^{a b} r_{, a} r_{, b}\right)$, where $r$ is the area radius.
} 


\subsection{Static vacuum æther}

The solution outside a static star is the unique vacuum solution for a given mass in which the æther is aligned with the Killing vector [31]. This "static æther" vacuum solution depends on the $c_{i}$ only through the combination $c_{14}$, and was found analytically (up to inversion of a transcendental equation) [31]. It has a globally timelike Killing vector, and is asymptotically flat. The spatial slices have a minimal area 2-sphere, inside of which the 2-spheres flare out to infinite area at a singularity on a would-be Killing horizon with vanishing surface gravity. The affine parameter distance to the singularity is finite along radial null geodesics. (The minimal two sphere is a property of the vacuum solution only; it never occurs in the presence of a matter source.) Although this static wormhole indicates the presence of a negative effective energy density in the field equation (i.e. the Einstein tensor has this property), all solutions in this family have positive total mass. In Ref. [34] it was found that this static æther solution is stable to linear perturbations under the same conditions as for flat spacetime, with the exception of the case $c_{123}=0$.

\subsection{Neutron stars}

The solution inside a fluid star has been found by numerical integration, both for constant density [31] and for realistic neutron star equations of state [35]. The maximum masses for neutron stars range from about 6 to $15 \%$ smaller than in GR when $c_{14}=1$, depending on the equation of state. The corresponding surface redshifts can be as much as $10 \%$ larger than in GR for the same mass. Measurements of high gravitational masses or precise surface redshifts thus have the potential to yield strong joint constraints on $c_{14}$ and the equation of state.

Sufficiently compact stars can have around them an innermost stable circular orbit (ISCO), which occurs in the Schwarzscild solution of GR at the radius $6 G_{N} M$. The ISCO in the static æther solution lies at a radius larger by approximately the factor $\left(1+0.03 c_{14}\right)$, with orbital frequency lower by the factor $\left(1-0.04 c_{14}\right)$. These small differences would likely be difficult to measure in practice.

\subsection{Black holes}

For black holes the æther cannot be aligned with the Killing vector, since the latter is not timelike on and inside the horizon. Instead, the æther is at rest at spatial infinity and flows inward at finite radii. The condition of regularity at the spin- 0 horizon (where the outgoing spin-0 waves propagate at constant radius) selects a unique solution from the one-parameter family of spherical stationary solutions for a given mass [36]. This is because the coefficient of the second radial derivative term in a field equation vanishes at the spin-0 horizon, which forces the second derivative to blow up unless the rest of the terms also vanish. Generically this regularity condition does not hold, but the solution can be tuned to satisfy it. When a black hole forms from collapse of matter, the spin-0 horizon develops in a nonsingular region of spacetime, where the evolution should be regular. This motivated the conjecture that collapse produces a black hole with nonsingular spin0 horizon, which has been confirmed for some particular examples in numerical simulations of collapse of a scalar field [37].

The black holes with nonsingular spin-0 horizons are rather close to Schwarzschild outside the horizon for a wide range of couplings; for instance, the ISCO radius differs by a factor $(1+$ 
$\left.0.043 c_{1}+0.061 c_{1}^{2}\right)$, in the case with $c_{3}=c_{4}=0$ and $c_{2}$ fixed so that the spin- 0 speed is unity [38]. (This expansion is accurate at least when $c_{1} \leq 0.5$. No solution with regular spin-0 horizon exists in this case when $c_{1} \gtrsim 0.8$.) Inside the horizon the solutions differ more, but like Schwarzschild they contain a spacelike singularity. Black hole solutions with singular spin-0 horizons have been studied in Ref. [39]. These solutions can differ much more outside the horizon. Quasi-normal modes of black holes in æ-theory have been investigated in Refs. [40].

\section{Special values of $c_{i}$}

It would be interesting if there existed a symmetry of the action restricting the values of the coupling parameters $c_{i}$, but none has been identified. Values for which the PPN parameters $\alpha_{1,2}$ vanish evidently lead to more symmetry in the weak field limit, but this does not appear to extend to a symmetry of the full theory. A microscopic theory might predict a particular relation or hierarchy between the coefficients, but no such theory is in hand. Hence any prior imposition of restrictions would be unjustified. Nevertheless, we may ask from a purely phenomenological perspective whether some simplifying restrictions could be compatible with observations. The answer is negative. It appears that, unless all of the parameters are much smaller than unity, then in order to be compatible with all of the theoretical and observational constraints (leaving aside the radiation reaction and strong self-field effects), most likely none of the $c_{i}$, nor $c_{13}, c_{14}$, or $c_{123}$ can vanish. We now discuss these special cases in some detail.

- The first case to be examined in detail $[8,7]$ was $c_{13}=c_{2}=c_{4}=0$, i.e. the "Maxwell action" (with the unit constraint on the vector). The PPN result for $\alpha_{2}$ (2.3) is infinite in this case, and the spin- 0 mode speed is zero. The perturbation series used in the PPN analysis is thus evidently not applicable. Independently of that, however, other problems with this case have been identified, such as the formation of shock discontinuities [7, 41] and a possibly related instability [34]. This case is equivalent to Maxwell theory in a special gauge if it is further restricted to the sector in which the Lagrange multiplier field vanishes (see [42, 7, 43, 44] and references therein). However, this equivalence holds only if one abandons the notion that the vector is itself physical. The shock discontinuities and instabilities of the vector can then be regarded as gauge artifacts, but the theory is no longer that of a Lorentz-violating vector field.

- The mode speeds (4.1) are all equal to unity if $c_{13}=c_{4}=0$ and $c_{2}=c_{1} /\left(1-2 c_{1}\right)$. In this case the PPN parameters become $\alpha_{1}=-4 c_{1}$ and $\alpha_{2}=0$, so the observational constraint on $\alpha_{1}$ imposes the stringent condition $\left|c_{1}\right| \lesssim 2.5 \times 10^{-5}$, and the other parameters are then similarly small. Positivity of the spin- 1 and spin- 0 mode energies imposes $0<c_{1}<1$. With small positive $c_{1}$, the remaining constraints can be satisfied as well.

- The case $c_{1}=c_{3}=0$ is evidently not covered by the existing PPN analysis, since $\alpha_{1}$ (2.2) is indeterminate and the spin- 1 mode speed is zero. (If $c_{2}=0$ then also the spin- 0 speed vanishes. If $c_{4}=0$ then the spin- 1 speed is indeterminate and the spin- 0 speed is infinite, but the energy of both modes is zero.) It appears that the post-Newtonian order of some field quantities must be modified in this case, which could be interesting to examine ab initio. 
- Assuming that (i) $\alpha_{1,2}=0$, (ii) the speed constraints $(4.10,4.11)$ are satisfied, (iii) the spin-1 mode speed (4.2) is not infinite with finite energy, and (iv) putting aside the case $c_{1}=c_{3}=0$ which is not covered by existing PPN analyses, the only viable case in which any of $c_{i}, c_{13}$, $c_{14}$, or $c_{123}$ vanishes is the special case $c_{3}=c_{4}=2 c_{1}+3 c_{2}=0$, with $2 / 3<c_{1}<1$. This large value of $c_{1}$ is probably inconsistent with the strong field constraints from orbital binaries, but as mentioned above those are not yet precisely known because the sensitivity parameters have not yet been computed for neutron stars, so this case is not yet ruled out. All the other special cases contradict one or more of the conditions (i)-(iv), which can be seen as follows. We halt when a case is reduced to a previous case.

$-c_{1}=0: \alpha_{1}=0$ then implies also $c_{3}=0$. As stated above, the case $c_{1}=c_{3}=0$ requires a new analysis.

$-c_{13}=0$ : The speed constraint (4.11) then implies also $c_{-}=0$, so $c_{1}=c_{3}=0$.

- $c_{14}=0: \alpha_{1}=0$ then implies $c_{3}^{2}=c_{1}^{2}$, so either $c_{+}=0$ or $c_{-}=0$. In either case, the spin-1 speed diverges unless $c_{1}=0$.

$-c_{123}=0$ : The denominator of the second term in $\alpha_{2}$ vanishes (2.3), so either $\alpha_{2}$ diverges or, if the numerator also vanishes, it is indeterminate. When $\alpha_{1}=0, \alpha_{2}$ becomes

$$
\alpha_{2}=-\frac{c_{13}^{2}\left(3 c_{1} c_{123}-c_{13}^{2}\right)}{c_{1}^{2} c_{123}\left(2-c_{14}\right)},
$$

so the numerator vanishes in this case only if $c_{13}=0$. (This also implies $c_{14}=0$.)

$-c_{2}=0: \alpha_{1,2}=0$ implies (2.4), which can be written as

$$
c_{2}=-c_{+}\left(c_{+}+3 c_{-}\right) / 6 c_{1} .
$$

Thus either $c_{+}=0$ (covered above) or $c_{+}+3 c_{-}=0$. In the latter case, either $c_{+}$or $c_{-}$ is negative, violating the speed constraints.

- $c_{3}=0: \alpha_{1}=0$ implies $c_{1}=0$ (covered above) or $c_{4}=0$. In the latter case, (2.4) or (8.2) then implies that $2 c_{1}+3 c_{2}=0$.

$-c_{4}=0: \alpha_{1}=0$ then implies $c_{3}=0$.

\section{Frontiers}

A number of questions remain open regarding phenomenology of æ⿱一𫝀-theory, as well as more fundamental issues. In this section some of these are briefly outlined.

- The most pressing issue is to compute the sensitivity parameters for neutron stars and black holes, so that strong field effects on radiation damping rates, waveforms, and post-Newtonian dynamics can be evaluated. To determine the sensitivities for a given compact object one might compute (presumably numerically) the exact solution for the object moving at some velocity with respect to the asymptotic aether, and match e.g. multipole moments of the asymptotic fields to those of the corresponding solution derived from the worldline action (6.1). It may be adequate to solve for linear perturbations of the exact, static solutions, rather than finding the exact moving solutions, but this may not be any more convenient. 
- Spherical collapse of a star to a black hole would be accompanied by a pulse of spin-0 radiation, since the æther must adjust from the static configuration (parallel to the Killing vector) to the black hole configuration (flowing across the horizon). This radiation may be much stronger than that of ordinary gravitational waves arising from asphericity of supernova core collapse. Moreover, it could in principle be measured by gravitational wave detectors, as seen from the polarization components (4.6).

- Rapidly rotating black holes, unlike the non-rotating ones, might turn out to be very different from the Kerr metrics of GR. If so, this could provide an interesting observational signature to detect or constrain. It may be that the easiest way to determine such solutions would be by numerical simulation of axisymmetric collapse.

- Although the linear perturbations all have positive energy for coupling parameters within in a certain range, the total, nonlinear energy has not been shown to be always positive in this range. (The positive energy theorem of GR does not apply since the æther stress tensor does not satisfy the dominant energy condition.) Even a spherically symmetric result in this regard would be useful. The question of energy positivity was addressed in Ref. [41], but attention was restricted there to the case where the coupling to gravity is neglected, and only some special cases were examined.

- Supersymmetry could potentially allow for a naturally suppressed Lorentz violation in the matter sector [45]. To include gravity in such a scheme would require a Lorentz violating supergravity. Can æ-theory be supersymmetrized? The argument [46] that supergravity has positive energy and therefore so must GR would presumably extend to æ-theory. Therefore, supersymmetrizability is likely only possible at most for those parameter ranges for which æ-theory has positive energy.

- Black hole thermodynamics remains a puzzle in æ-theory. It has not been possible to identify the entropy and a corresponding "first law" of black hole mechanics, despite the existence of an identity relating mass and angular momentum variations to a horizon integral [32]. The difficulty is related to the fact that the æther necessarily diverges at the bifurcation surface, so the methods usually employed [47] are not applicable. One might expect that the problem is related to the fact that, in a theory with multiple limiting speeds, no unique horizon is selected. However, even for the cases mentioned in section 8 for which the mode speeds are all equal to unity, the entropy has not yet been identified.

A further important issue is the status of the generalized second law of thermodynamics. It has been argued that in a theory with more than one limiting speed, both quantum Hawking radiation [48] and classical energy extraction [49] methods can be exploited to violate the generalized second law of thermodynamics. This suggests that there may be some deep problem with such theories, including ae-theory (with unequal mode speeds).

- Lorentz-violating matter fields can be consistently coupled to gravity using æ-theory. In this way gravitational back-reaction of such fields is well defined. This has been studied in a cosmological setting [50], and the issue of renormalization of the corresponding quantum 
stress tensor has recently been seriously tackled [51]. Counter-terms renormalizing the $æ-$ theory action are generally required. Such analyses should enable the quantum back reaction to cosmological expansion as well as to Hawking radiation to be studied.

- Quantization of ae-theory may be instructive to investigate. The dynamical unit timelike vector field determines a time flow with respect to which evolution can be referred. This "material reference system" could perhaps be employed $[52,53]$ to address the "problem of time" $[54,55]$ in canonical quantum gravity.

- Vacuum ae-theory in 1+1 dimensions might provide a useful simplified model for studying quantum effects. The classical theory has been investigated [56], where the full solution space was mapped out. The solutions include de Sitter and anti-de Sitter spacetimes, with a uniformly accelerated æther that is invariant under a two-dimensional subgroup of $\mathrm{SO}(2,1)$ generated by a boost and a null rotation. (The æther becomes infinitely stretched on certain Killing horizons in these solutions.) The only other solution is a peculiar one with singularities and no Killing vectors.

\section{Conclusion}

Einstein-æther theory provides a theoretical laboratory in which gravitational effects of possible Lorentz violation can be meaningfully studied without abandoning the generally covariant framework of general relativity. It is striking that æ-theory can match observations to the degree that it can. While of course starting with four free parameters $c_{1,2,3,4}$ makes this easier, the freedom to choose these parameters might have already been used up in setting the PPN parameters to agree with those of GR. Instead, only two parameters need be fixed at that stage, leaving $c_{1}$ and $c_{3}$ free. Then, despite possessing three distinct types of linearized wave modes, all positive energy, stability and vacuum Čerenkov requirements on the modes are met within a large common region of the $\left(c_{1}, c_{3}\right)$ space. Within this same parameter space, the dynamics of the cosmological scale factor and perturbations differ little from GR, and non-rotating neutron star and black hole solutions are quite close to those of GR (but may be distinguishable with future observations).

Additional constraints arise from the observed radiation damping rate in binaries. For systems with weak self-fields, a constraint of order $10^{-3}$ would be imposed on one combination of the two parameters $c_{1}$ and $c_{3}$. Current observations involve pulsars which possess strong self-fields. Presuming that the sensitivity parameters for neutron stars turn out to have the expected magnitude, such binaries will exhibit effects constraining both the parameters to be less than around $10^{-2}$, in addition to the stronger radiation damping constraint on one combination. It may turn out that observations of multiple systems with different combinations of sensitivities will constrain both parameters separately at the $10^{-3}$ level.

\section{Acknowledgments}

I am grateful to C.T. Eling, B.Z. Foster, B. Li, E. Lim, D. Mattingly, I. Rothstein and T. Sotiriou for helpful discussions and comments on drafts of this article. This work was supported by NSF grant PHY-0601800. 


\section{References}

[1] D. Mattingly, “Modern tests of Lorentz invariance,” Living Rev. Rel. 8, 5 (2005) [arXiv:gr-qc/0502097].

[2] C. Eling and T. Jacobson, "Static post-Newtonian equivalence of GR and gravity with a dynamical preferred frame,” Phys. Rev. D 69, 064005 (2004) [arXiv:gr-qc/0310044].

[3] C. P. Burgess, "Quantum gravity in everyday life: General relativity as an effective field theory," Living Rev. Rel. 7, 5 (2004) [arXiv:gr-qc/0311082].

[4] C.M. Will and K. Nordvedt, Jr., "Conservation laws and preferred frames in relativistic gravity. I. Preferred frame theories and an extended PPN formalism," Astrophys. J. 177, 757 (1972);

K. Nordvedt, Jr. and C.M. Will, "Conservation laws and preferred frames in relativistic gravity. II. Experimental evidence to rule out preferred frame theories of gravity," Astrophys. J. 177, 775 (1972); R.W. Hellings and K. Nordvedt, Jr., "Vector-metric theory of gravity," Phys. Rev. D7, 3593 (1973).

[5] J. W. Elliott, G. D. Moore and H. Stoica, "Constraining the new æther: Gravitational Cherenkov radiation,” JHEP 0508, 066 (2005) [arXiv:hep-ph/0505211].

[6] See, for example, M. Gasperini, "Singularity prevention and broken Lorentz symmetry," Class. Quantum Grav. 4, 485 (1987); "Repulsive gravity in the very early Universe," Gen. Rel. Grav. 30, 1703 (1998); and references therein.

[7] T. Jacobson and D. Mattingly, “Gravity with a dynamical preferred frame," Phys. Rev. D 64, 024028 (2001) [arXiv:gr-qc/0007031].

[8] V. A. Kostelecky and S. Samuel, "Gravitational Phenomenology In Higher Dimensional Theories And Strings,” Phys. Rev. D 40, 1886 (1989).

[9] B. M. Gripaios, "Modified gravity via spontaneous symmetry breaking," JHEP 0410, 069 (2004) [arXiv:hep-th/0408127].

[10] T. G. Zlosnik, P. G. Ferreira and G. D. Starkman, "Modifying gravity with the æther: An alternative to dark matter,” Phys. Rev. D 75, 044017 (2007) [arXiv:astro-ph/0607411].

[11] H. Zhao, "Coincidences of Dark Energy with Dark Matter - Clues for a Simple Alternative?," arXiv:0710.3616 [astro-ph].

[12] S. Kanno and J. Soda, "Lorentz violating inflation,” Phys. Rev. D 74, 063505 (2006) [arXiv:hep-th/0604192].

[13] J. D. Bekenstein, "Relativistic gravitation theory for the MOND paradigm," Phys. Rev. D 70, 083509 (2004) [Erratum-ibid. D 71, 069901 (2005)] [arXiv:astro-ph/0403694].

[14] J. W. Moffat, "Scalar-tensor-vector gravity theory," JCAP 0603, 004 (2006) [arXiv:gr-qc/0506021].

[15] R. Bluhm and V. A. Kostelecky, "Spontaneous Lorentz violation, Nambu-Goldstone modes, and gravity,” Phys. Rev. D 71, 065008 (2005) [arXiv:hep-th/0412320].

[16] B. Z. Foster, "Metric redefinitions in Einstein-æther theory," Phys. Rev. D 72, 044017 (2005) [arXiv:gr-qc/0502066].

[17] J. F. Barbero G. and E. J. S. Villasenor, “Lorentz Violations and Euclidean Signature Metrics,” Phys. Rev. D 68, 087501 (2003) [arXiv:gr-qc/0307066].

[18] S. M. Carroll and E. A. Lim, "Lorentz-violating vector fields slow the universe down," Phys. Rev. D 70, 123525 (2004) [arXiv:hep-th/0407149]. 
[19] C. M. Will, "The Confrontation between General Relativity and Experiment," Living Reviews in Relativity 9, 3 (2006).

[20] B. Z. Foster and T. Jacobson, "Post-Newtonian parameters and constraints on Einstein-æther theory," Phys. Rev. D 73, 064015 (2006) [arXiv:gr-qc/0509083].

[21] M. L. Graesser, A. Jenkins and M. B. Wise, "Spontaneous Lorentz violation and the long-range gravitational preferred-frame effect,” Phys. Lett. B 613, 5 (2005) [arXiv:hep-th/0501223].

[22] B. Z. Foster, "Radiation damping in Einstein-æther theory," Phys. Rev. D 73, 104012 (2006) [arXiv:gr-qc/0602004].

[23] D. Mattingly and T. Jacobson, "Relativistic gravity with a dynamical preferred frame," in Proceedings of the Second Meeting on CPT and Lorentz Symmetry, Ed. V. Alan Kostelecky (Singapore, World Scientific, 2002) [arXiv:gr-qc/0112012].

[24] T. Jacobson and D. Mattingly, “Einstein-Aether waves,” Phys. Rev. D 70, 024003 (2004) [arXiv:gr-qc/0402005].

[25] C. Eling, "Energy in the Einstein-æther theory,” Phys. Rev. D 73, 084026 (2006) [arXiv:gr-qc/0507059].

[26] E. A. Lim, “Can We See Lorentz-Violating Vector Fields in the CMB?,” Phys. Rev. D 71, 063504 (2005) [arXiv:astro-ph/0407437].

[27] B. Li, D. F. Mota and J. D. Barrow, "Detecting a Lorentz-Violating Field in Cosmology," arXiv:0709.4581 [astro-ph].

[28] B. Z. Foster, "Strong field effects on binary systems in Einstein-æther theory," arXiv:0706.0704 [gr-qc].

[29] D. M. Eardley, "Observable effects of a scalar gravitational field in a binary pulsar," Astrophysical Journal 196, L59 (1975).

[30] B. Z. Foster, private communication.

[31] C. Eling and T. Jacobson, "Spherical Solutions in Einstein-Aether Theory: Static Aether and Stars," Class. Quant. Grav. 23, 5625 (2006) [arXiv:gr-qc/0603058].

[32] B. Z. Foster, "Noether charges and black hole mechanics in Einstein-æther theory," Phys. Rev. D 73, 024005 (2006) [arXiv:gr-qc/0509121].

[33] S. A. Hayward, “Gravitational energy in spherical symmetry,” Phys. Rev. D 53, 1938 (1996) [arXiv:gr-qc/9408002].

[34] M. D. Seifert, "Stability of spherically symmetric solutions in modified theories of gravity," Phys. Rev. D 76, 064002 (2007) [arXiv:gr-qc/0703060].

[35] C. Eling, T. Jacobson and M. Coleman Miller, "Neutron stars in Einstein-æther theory," Phys. Rev. D 76, 042003 (2007) [arXiv:0705.1565 [gr-qc]].

[36] C. Eling and T. Jacobson, "Black holes in Einstein-æther theory," Class. Quant. Grav. 23, 5643 (2006) [arXiv:gr-qc/0604088].

[37] D. Garfinkle, C. Eling and T. Jacobson, "Numerical simulations of gravitational collapse in Einstein-æther theory,” Phys. Rev. D 76, 024003 (2007) [arXiv:gr-qc/0703093].

[38] C. T. Eling, private communication. 
[39] T. Tamaki and U. Miyamoto, "Generic features of Einstein-Aether black holes," arXiv:0709.1011 [gr-qc].

[40] R. A. Konoplya and A. Zhidenko, "Perturbations and quasi-normal modes of black holes in Einstein-æther theory,” Phys. Lett. B 644, 186 (2007) [arXiv:gr-qc/0605082]; "Gravitational spectrum of black holes in the Einstein-Aether theory,” Phys. Lett. B 648, 236 (2007) [arXiv:hep-th/0611226].

[41] M. A. Clayton, "Causality, shocks and instabilities in vector field models of Lorentz symmetry breaking," arXiv:gr-qc/0104103.

[42] Y. Nambu, "Quantum electrodynamics in nonlinear gauge," Prog. Theor. Phys. Suppl. Extra No. (1968) 190-195

[43] J. L. Chkareuli, C. D. Froggatt, J. G. Jejelava and H. B. Nielsen, “Constrained Gauge Fields from Spontaneous Lorentz Violation," arXiv:0710.3479 [hep-th].

[44] R. Bluhm, S. H. Fung and A. Kostelecky, "Spontaneous Lorentz and Diffeomorphism Violation, Massive Modes, and Gravity,” arXiv:0712.4119 [hep-th].

[45] S. Groot Nibbelink and M. Pospelov, "Lorentz violation in supersymmetric field theories," Phys. Rev. Lett. 94, 081601 (2005) [arXiv:hep-ph/0404271]; P. A. Bolokhov, S. G. Nibbelink and M. Pospelov, "Lorentz violating supersymmetric quantum electrodynamics," Phys. Rev. D 72, 015013 (2005) [arXiv:hep-ph/0505029].

[46] S. Deser and C. Teitelboim, "Supergravity Has Positive Energy,” Phys. Rev. Lett. 39, 249 (1977); M. T. Grisaru, "Positivity Of The Energy In Einstein Theory,” Phys. Lett. B 73, 207 (1978); E. Witten, "A Simple Proof Of The Positive Energy Theorem," Commun. Math. Phys. 80, 381 (1981).

[47] R. M. Wald, "Black hole entropy is the Noether charge," Phys. Rev. D 48, 3427 (1993) [arXiv:gr-qc/9307038]; T. Jacobson, G. Kang and R. C. Myers, "On Black Hole Entropy,” Phys. Rev. D 49, 6587 (1994) [arXiv:gr-qc/9312023]; V. Iyer and R. M. Wald, "Some properties of Noether charge and a proposal for dynamical black hole entropy,” Phys. Rev. D 50, 846 (1994) [arXiv:gr-qc/9403028].

[48] S. L. Dubovsky and S. M. Sibiryakov, "Spontaneous breaking of Lorentz invariance, black holes and perpetuum mobile of the 2nd kind,” Phys. Lett. B 638, 509 (2006) [arXiv:hep-th/0603158].

[49] C. Eling, B. Z. Foster, T. Jacobson and A. C. Wall, "Lorentz violation and perpetual motion,” Phys. Rev. D 75, 101502 (2007) [arXiv:hep-th/0702124].

[50] T. Jacobson and D. Mattingly, "Generally covariant model of a scalar field with high frequency dispersion and the cosmological horizon problem," Phys. Rev. D 63, 041502 (2001)

[arXiv:hep-th/0009052]; M. Lemoine, M. Lubo, J. Martin and J. P. Uzan, “The stress-energy tensor for trans-Planckian cosmology," Phys. Rev. D 65, 023510 (2002) [arXiv:hep-th/0109128];

R. H. Brandenberger and J. Martin, "Back-reaction and the trans-Planckian problem of inflation revisited," Phys. Rev. D 71, 023504 (2005) [arXiv:hep-th/0410223].

[51] D. Lopez Nacir and F. D. Mazzitelli, "Backreaction in trans-Planckian cosmology: renormalization, trace anomaly and selfconsistent solutions,” Phys. Rev. D 76, 024013 (2007) [arXiv:0706.2179 [gr-qc]]; D. L. Nacir and F. D. Mazzitelli, "New counterterms induced by trans-Planckian physics in semiclassical gravity," arXiv:0711.4554 [gr-qc]; M. Rinaldi, “A momentum-space representation of Green's functions with modified dispersion on ultra-static space-time," Phys. Rev. D 76, 104027 (2007) [arXiv:0709.2657 [gr-qc]].

[52] J. D. Brown and K. V. Kuchar, "Dust as a standard of space and time in canonical quantum gravity," Phys. Rev. D 51, 5600 (1995) [arXiv:gr-qc/9409001]. 
[53] J. D. Brown and D. Marolf, "On Relativistic Material Reference Systems," Phys. Rev. D 53, 1835 (1996) [arXiv:gr-qc/9509026].

[54] C. J. Isham, "Canonical quantum gravity and the problem of time," arXiv:gr-qc/9210011.

[55] K. Kuchar, "The problem of time in quantum geometrodynamics," in The Arguments of Time, Ed. J. Butterfield (Oxford University Press, 2006) pp.169-195

[56] C. Eling and T. Jacobson, "Two-dimensional gravity with a dynamical æther," Phys. Rev. D 74, 084027 (2006) [arXiv:gr-qc/0608052]. 